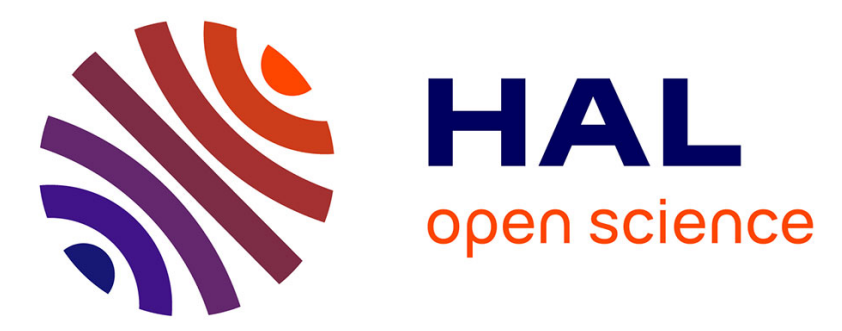

\title{
Homogenization methods for interface modeling in damaged masonry
}

\author{
Amna Rekik, Frédéric Lebon
}

\section{To cite this version:}

Amna Rekik, Frédéric Lebon. Homogenization methods for interface modeling in damaged masonry. Advances in Engineering Software, 2012, 46 (1), pp.35-42. 10.1016/j.advengsoft.2010.09.009 . hal00652805

\section{HAL Id: hal-00652805 https://hal.science/hal-00652805}

Submitted on 16 Dec 2011

HAL is a multi-disciplinary open access archive for the deposit and dissemination of scientific research documents, whether they are published or not. The documents may come from teaching and research institutions in France or abroad, or from public or private research centers.
L'archive ouverte pluridisciplinaire HAL, est destinée au dépôt et à la diffusion de documents scientifiques de niveau recherche, publiés ou non, émanant des établissements d'enseignement et de recherche français ou étrangers, des laboratoires publics ou privés. 


\title{
Homogenization methods for interface modeling in damaged masonry
}

\author{
A. Rekik ${ }^{1}$, F. Lebon ${ }^{2}$ \\ 1 Institut PRISME, Polytech'Orléans, 8 rue Léonard de Vinci, 45072 Orléans Cedex 2 \\ ${ }^{2}$ LMA CNRS UPR 7051, Université de Provence, 31, Chemin Joseph Aiguier, 13402 \\ Marseille Cedex 20
}

\begin{abstract}
The aim of the present study was to predict damage to masonry by combining structural analysis and homogenization methods. In the case of a masonry structure composed of bricks and mortar, a third material is assumed to exist, which is a mixture of the two materials sandwiched between the other two. This new layer has a small thickness, a low stiffness and a given damage ratio. The mechanical problem set by this masonry, which was initially a $3-\mathrm{D}$ problem, is solved numerically in $2-\mathrm{D}$ terms using finite element methods and modeling the three materials: brick, mortar and the interface material defined above. The properties of the third material are obtained by performing the following three steps: (i) first an exact homogenization of a brick/mortar laminate defining a first homogeneous equivalent medium (HEM-1) is performed. (ii) Secondly, we assume the HEM-1 to be damaged and apply the Kachanov model to assess the global behavior of the damaged HEM-1, thus defining a second equivalent homogeneous medium denoted HEM-2. (iii) Thirdly, asymptotic analysis is performed to model HEM-2 as an interface or a joint. The properties of this joint are deduced from those of the HEM-2 material. This interface is modeled numerically with connector finite elements.

This method is applied to two cases: a triplet of full bricks and a triplet of hollow bricks subjected twice to shear loading. The numerical results obtained are compared with experimental data available in the literature.
\end{abstract}

Key words: masonry structure, interface damage, homogenization, asymptotic analysis, finite element method. 


\section{Introduction}

Traditionally, as in ancient Greece and Egypt, people have always built their homes with natural materials available nearby, such as stones and bricks, in particular. Masonry is therefore one of the oldest construction materials. Masonry is still commonly used nowadays to build houses because of its qualities of strength, solidity, durability and fire resistance, and its elegant appearance, etc. However, masonry, which is not generally thought to be a highly technological material, shows highly complex behaviour, due in particular to the interactions between the components (mortar, bricks, concrete blocks and stones) and the anisotropy induced by the direction of the joints, which are a source of weakness. Masonry structures were classically designed on the basis of empirical rules. Modern virtual methods of design developed only quite recently. In particular, the structures built long ago were extremely stable because they were massive. In modern masonry buildings the walls are very thick for economic reasons and the stability has to be studied from the theoretical point of view, especially when wind or earthquakes are liable to occur. The strength of the masonry then becomes more critical and it is necessary to study the solidity of the structure using fine models and numerical simulations as in the case of concrete and steel structures. Other problems such as cracks are intolerable for the occupants of buildings, and preventing this problem also requires more detailed studies on the design of masonry structures.

In this paper, we deal with the resistance of masonry to in-plane shear forces. It is classically held that the seismic vulnerability of masonry buildings depends strongly on their resistance to shear forces. It is therefore of great interest to model and test the shear responses of building components subjected to loading of this kind, especially cyclic loading. These responses have generally been characterized by a peak load, loss of rigidity and energy dissipation. To summarize considerably, two methods of modeling masonry structures have been used so far.

The first method involves macroscopic models, in which the wall is assumed to be a single structural element characterized by a non linear response when it is exposed to shear forces $[1,2,3,4,5,6,7,37]$. These models, which are generally based on the use of homogenization techniques, usually take the properties of the materials and the bonding between the various components into account $[24,25,26,27,28,29,30,31,32,33,34,35]$.

On the other hand, some models have been developed for predicting the 
evolution of damage to the interface between two initially bonded deformable bodies $[8,9,10,11,12,13,14,15,16,17,18,19,20,21,36,38]$. These models are based mostly on phenomenological approaches. In this paper, an original multi-level model for interfaces based on homogenization techniques is presented, which takes the characteristics of the masonry into account.

The first part of this paper deals with the mechanical modeling approach used, and in particular with the model developed. The multi-level approach used is described. This approach takes the mechanical characteristics of the mortar and bricks, the presence of micro-cracks and the thickness of the interface into account. In the second part, the numerical procedure used and implemented using a finite element software program is presented and some numerical examples are given and compared with experimental data $[11,6]$. Details of the computations are given in appendices $\mathrm{A}$ and $\mathrm{B}$.

\section{The method of analysis proposed}

In order to model interface damage to masonry structures, the present method based on homogenization theories, asymptotic techniques and finite element methods was developed. The main steps involved in this method will be described below. Most studies on masonry structures have dealt with only two materials: brick and mortar. In the present work, we assume the existence of a third material: a mixture of brick and mortar with a crack density $\rho$. To obtain the effective properties of the damaged intermediate material, three steps are performed. First we calculate the exact effective properties of the material devoid of cracks using homogenization techniques for laminate composites, and thus define a first homogeneous equivalent medium, which will be referred below as HEM-1. In the second step, we attribute the crack density $\rho$ to the material HEM-1. To model the macroscopic behavior of the cracked material HEM-1, we use the Kachanov model and then define a new homogeneous equivalent medium HEM-2. This material sandwiched between the brick and mortar is taken to have a small thickness, and its mechanical behavior can therefore be obtained using asymptotic techniques to shift from the micro to macro level. With this interface law, the masonry structure problem can be solved using finite element methods.

The following overall scheme (Fig.1) describes the principles underlying the model proposed: 


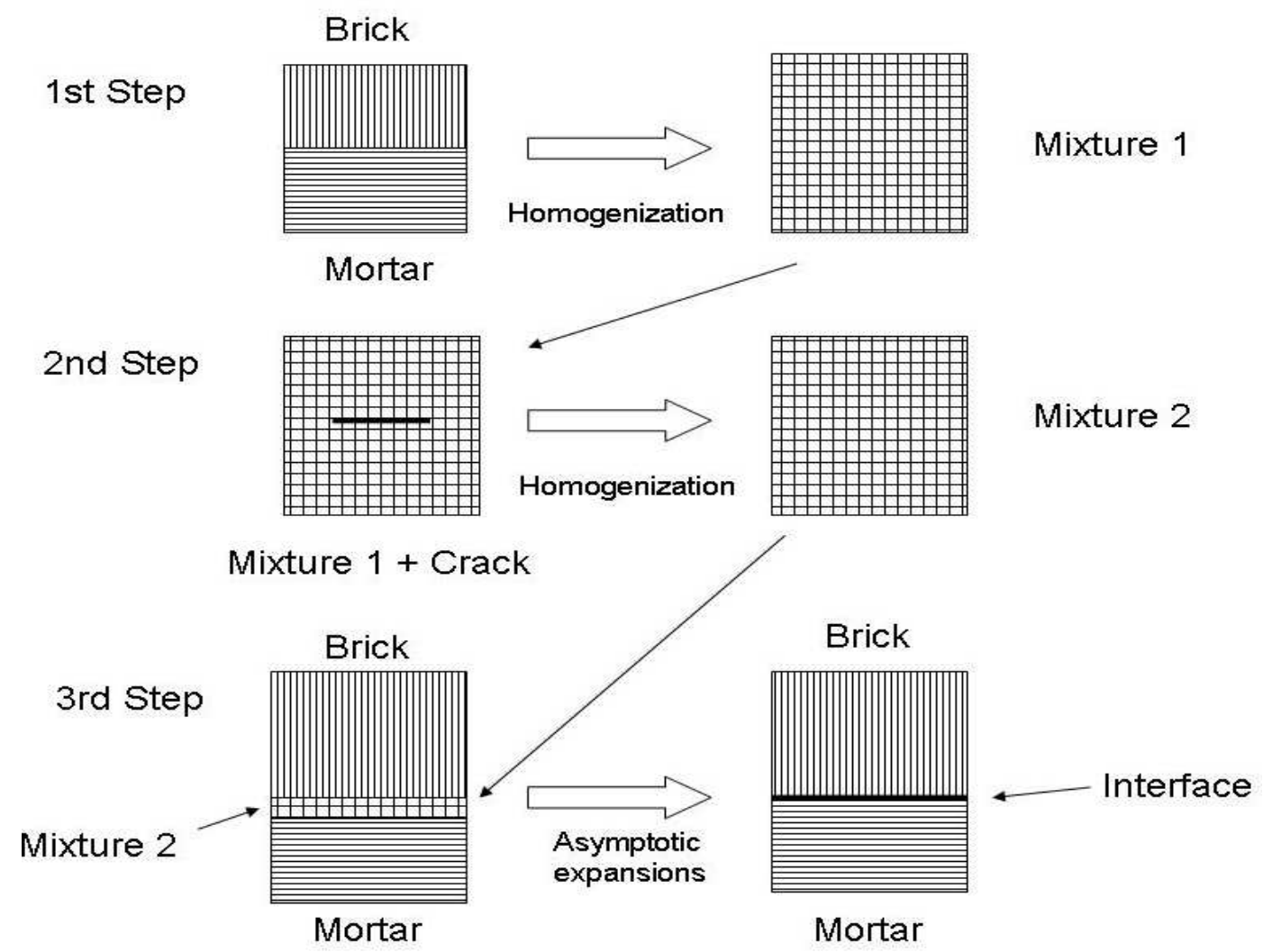

Figure 1: Principle of the proposed model

2.1. First step: undamaged stratified composite homogenization

The behavior of brick and mortar is assumed to be isotropic and linear, as described by the local law: 


$$
\varepsilon_{j k}^{i}=S_{j k l n}^{i} \sigma_{l n}^{i}, \quad \text { for } \quad j, k, l, n=1,2,3
$$

Where $S^{i}$ is the compliance tensor of the phase $i=b, m$. The indexes $b$ and $m$ denote the brick and mortar phases respectively. Each fourth-order local homogeneous phase compliance tensor $S^{i}$ reads: $S^{i}=\frac{1}{3 k^{i}} J+\frac{1}{2 \mu^{i}} K$, where the compressibility and the shear coefficients are given by: $k^{i}=\frac{E^{i}}{3\left(1-2 \nu^{i}\right)}$ and $\mu^{i}=\frac{E^{i}}{2\left(1+\nu^{i}\right)}$, respectively. $E^{i}$ and $\nu^{i}$ are the Young's modulus and the Poisson ratio, respectively, of each phase $i$. The fourth-order tensors $J$ and $K$ are given by the relations: $J=\frac{1}{3} i \otimes i$ and $K=I-J$, respectively, where $i$ is the second-order identity tensor and $I$ is the fourth-order one.

The macroscopic behavior law of the laminate brick/mortar reads:

$$
\bar{\varepsilon}=\tilde{S}^{0}: \bar{\sigma} \quad \text { where } \quad \bar{\varepsilon}=\sum_{i=b, m} f^{i} \varepsilon^{i}=\sum_{i=b, m} f^{i} S^{i}: \sigma^{i}
$$

where $f^{i}(i=b, m)$ denotes the volume fractions of the phases, $\tilde{S}^{0}$ is the effective fourth-order compliance tensor of the transversely isotropic crackfree laminate. According to the modified Voigt notation [23], the macroscopic law reads:

$$
\left(\begin{array}{r}
\bar{\varepsilon}_{11} \\
\bar{\varepsilon}_{22} \\
\bar{\varepsilon}_{33} \\
\sqrt{2} \bar{\varepsilon}_{23} \\
\sqrt{2} \bar{\varepsilon}_{13} \\
\sqrt{2} \bar{\varepsilon}_{12}
\end{array}\right)=\left(\begin{array}{rrrrrr}
\tilde{S}_{111}^{0} & \tilde{S}_{1122}^{0} & \tilde{S}_{1133}^{0} & 0 & 0 & 0 \\
\tilde{S}_{1122}^{0} & \tilde{S}_{2222}^{0} & \tilde{S}_{1133}^{0} & 0 & 0 & 0 \\
\tilde{S}_{1133}^{0} & \tilde{S}_{1133}^{0} & \tilde{S}_{3333}^{0} & 0 & 0 & 0 \\
0 & 0 & 0 & 2 \tilde{S}_{1313}^{0} & 0 & 0 \\
0 & 0 & 0 & 0 & 2 \tilde{S}_{1313}^{0} & 0 \\
0 & 0 & 0 & 0 & 0 & 2 \tilde{S}_{1212}^{0}
\end{array}\right)\left(\begin{array}{r}
\bar{\sigma}_{11} \\
\bar{\sigma}_{22} \\
\bar{\sigma}_{33} \\
\sqrt{2} \bar{\sigma}_{23} \\
\sqrt{2} \bar{\sigma}_{13} \\
\sqrt{2} \bar{\sigma}_{12}
\end{array}\right)
$$

The unknown components of the compliance tensor $\tilde{S}^{0}$ are determined in Appendix A by applying four independent loads: axial tension, axisymmetric loading, plane traction combined with a plane shear load and a longitudinal shear load. With each loading condition, it is necessary to fulfill two conditions at the brick/mortar interface as regards: (i) the continuity of the normal stress vector $\sigma . e_{3}$, i.e.:

$$
\sigma_{j 3}^{b}=\sigma_{j 3}^{m} \quad \text { for every index } j=1,2,3
$$


and (ii) the continuity of the in-plane deformation components

$$
\varepsilon_{k j}^{b}=\varepsilon_{k j}^{m} \quad \text { for the couplets } \quad(k, j)=(1,1),(2,2) \text { and }(1,2)
$$

In [39], the macroscopic description of a periodic stratified composite is obtained differently, using the asymptotic expansion technique [40] up to third order. The expression for the macroscopic elastic tensor $\tilde{C}^{0}=\left(\tilde{S}^{0}\right)^{-1}$ is written in terms of the elastic properties of the layers and its volume fractions in the periodic cell.

\subsection{Second step: homogenization of the damaged composite}

In this section, the material HEM-1 is assumed to contain an arbitrary crack orientation distribution. Kachanov and Mauge [15] provided an accurate approximation of the effective behaviour of the cracked material HEM-1. Assuming that cracks are rectilinear and located in the plane $\left(e_{1}, e_{3}\right)$, the initial $3-\mathrm{D}$ problem can be reduced to a $2-\mathrm{D}$ problem. In this case, it has been established [15] that the overall strain $\bar{\varepsilon}$ in a solid with $N$ cracks can be written in the form

$$
\bar{\varepsilon}=\tilde{S}: \bar{\sigma}=\tilde{S}^{0}: \bar{\sigma}+\frac{1}{A} \sum_{k=1}^{N}(n \otimes<b>+<b>\otimes n)^{(k)} l^{(k)}=\left(\tilde{S}^{0}+\Delta \tilde{S}\right): \bar{\sigma}
$$

where $\tilde{S}$ is the effective compliance of the cracked material, $\tilde{S}^{0}$ is the compliance of the crack-free matrix, $n^{(k)}, 2 l^{(k)}$ and $\langle b\rangle^{(k)}$ are the normal unit vector, the length of the $k$ th crack and the average displacement discontinuity (COD) vector at the $k$ th crack; and $A$ is the representative averaging area.

The potential of the cracked material is therefore the sum of two terms:

$$
f(\bar{\sigma})=\frac{1}{2} \bar{\varepsilon}: \tilde{S}: \bar{\varepsilon}=\frac{1}{2} \bar{\sigma}: \tilde{S}^{0}: \bar{\sigma}+\frac{1}{A} \sum_{k=1}^{N}(n \cdot \bar{\sigma} .<b>)^{(k)} l^{(k)}=f^{0}(\bar{\sigma})+\Delta f
$$

$f^{0}$ is the potential of the crack-free material and $\Delta f$ is the change of $f$ due to the presence of cracks.

The problem of finding the additional compliance $\Delta \tilde{S}$ (relation (6)) due to cracks is reduced to finding the COD tensor $B$ in terms of the average stress $\bar{\sigma}$. The latter is defined by the relation $[16,17]$ :

$$
<b>=n \cdot \bar{\sigma} \cdot B
$$


The components of $B$ depend on the compliance matrix $\tilde{S}^{0}$ and the orientation of the crack with respect to the matrix anisotropy axes. In the crack coordinate system $(t, n)$, where $t$ is a unit vector which is tangential to the crack so that:

$$
\begin{gathered}
t=\cos \phi e_{1}+\sin \phi e_{3} \\
n=-\sin \phi e_{1}+\cos \phi e_{3} \\
B=B_{n n} n \otimes n+B_{t t} t \otimes t+B_{n t} n \otimes t+B_{t n} t \otimes n
\end{gathered}
$$

$\left(e_{1}, e_{3}\right)$ are the principal axes of the matrix orthotropy and $\phi$ is the angle between the crack line and the $e_{1}$-principal axis.

The expressions for the COD tensor (7) and (8) are used to obtain the change in $f$ due to the presence of a family of non-interacting cracks for which $t$ and $n$ are the same:

$$
\Delta f=\rho \bar{\sigma}: n \cdot\left\{B_{n n} n \otimes n+B_{t t} t \otimes t+B_{n t}(n \otimes t+t \otimes n)\right\} \cdot n: \bar{\sigma}
$$

where $\rho=\frac{1}{A} \sum_{k}\left(l^{(k)}\right)^{2}$ is the conventional scalar crack density parameter. In the considered case of an orthotropic matrix with one arbitrarily oriented family of parallel cracks $\left(-\frac{\pi}{2} \leq \phi \leq \frac{\pi}{2}\right), B$ can be expressed in the following form, using the solution given by Lekhnitsky [19] and Savin [20].

$$
\left\{\begin{array}{l}
B_{t t}=C(1-D \cos 2 \phi) l \\
B_{n n}=C(1+D \cos 2 \phi) l \\
B_{t n}=C D(\sin 2 \phi) l
\end{array}\right.
$$

where

$$
\left\{\begin{array}{l}
C=\frac{\pi}{4} \frac{\sqrt{E_{1}^{0}}+\sqrt{E_{3}^{0}}}{\sqrt{E_{1}^{0} E_{3}^{0}}}\left(\frac{1}{G_{13}^{0}}-2 \frac{\nu_{13}^{0}}{E_{1}^{0}}+\frac{2}{\sqrt{E_{1}^{0} E_{3}^{0}}}\right)^{\frac{1}{2}} \\
D=\frac{\sqrt{E_{1}^{0}}-\sqrt{E_{3}^{0}}}{\sqrt{E_{1}^{0}}+\sqrt{E_{3}^{0}}}
\end{array}\right.
$$

$E_{1}^{0}, E_{3}^{0}, \nu_{13}^{0}$ and $G_{13}^{0}$ are the elastic engineering constants of the crack-free material HEM-1. These constants are deduced from the effective elastic 
compliances $\tilde{S}^{0}[19]$ as follows:

$$
\left\{\begin{array}{l}
\tilde{S}_{1111}^{0}=\frac{1}{E_{1}^{0}} \\
\tilde{S}_{3333}^{0}=\frac{1}{E_{3}^{0}} \\
\tilde{S}_{1133}^{0}=-\frac{\nu_{13}^{0}}{E_{1}^{0}} \\
\tilde{S}_{1313}^{0}=\frac{1}{G_{13}^{0}}
\end{array}\right.
$$

We note here the dependance of the sign of $D$ on the anisotropy ratio $E_{1}^{0} / E_{3}^{0}$.

Substituting $B$ from (10) into (9), we obtain the elastic potential $f$, and therefore, all the moduli of the material HEM-2 (i.e. HEM-1 with cracks). On the principal axes $e_{1}, e_{3}$, the effective properties of HEM-2 are therefore given by:

$$
\left\{\begin{array}{l}
\frac{E_{1}}{E_{1}^{0}}=\frac{1}{1+2 \rho \sin ^{2} \phi\left(B_{t t} \cos ^{2} \phi+B_{n n} \sin ^{2} \phi-B_{n t} \sin 2 \phi\right) E_{1}^{0}} \\
\frac{E_{3}}{E_{3}^{0}}=\frac{1}{1+2 \rho \cos ^{2} \phi\left(B_{t t} \sin ^{2} \phi+B_{n n} \cos ^{2} \phi+B_{n t} \sin 2 \phi\right) E_{3}^{0}} \\
\frac{G_{13}}{G_{13}^{0}}=\frac{1}{1+\rho\left(B_{n n} \sin ^{2} 2 \phi+B_{t t} \cos ^{2} 2 \phi-B_{n t} \sin 4 \phi\right) G_{13}^{0}} \\
\frac{\nu_{13}}{E_{1}}=\frac{\nu_{13}^{0}}{E_{1}^{0}}
\end{array}\right.
$$

The stiffness tensor $\tilde{C}$ of the material HEM-2 is obtained by performing inversion on the compliance tensor $\tilde{S}$ deduced from the elastic engineering constants $E_{1}, E_{3}, \nu_{13}$ and $G_{13}$ (giving a relation similar to (12)). For the following applications, we assume the cracks to be parallel to the $e_{1}$-principal axis, i.e., $\phi=0$. In this case, the engineering constants defining the behavior of HEM-2 read:

$$
E_{1}=E_{1}^{0}, E_{3}=E_{3}^{0} /\left(1+2 \rho B_{n n} E_{3}^{0}\right), G_{13}=G_{13}^{0} /\left(1+\rho B_{t t} G_{13}^{0}\right) \text { and } \nu_{13}=\nu_{13}^{0}
$$

where, in this case, $B_{n n}=C(1+D) l$ and $B_{t t}=C(1-D) l$.

\subsection{Third step: asymptotic analysis and interface law}

In this section, we consider a thin joint composed of the material defined above, which is sandwiched between brick and mortar. Since the joint is 
thin and soft, it is natural to use asymptotic techniques, to study the limit problem (when the thickness is equal to zero) and to replace the joint by an interface law defined along the limit surface. We take $\varepsilon$ to denote the thickness of the joint, which is assumed to be constant and $S$ to denote the limit surface of the joint (a line in 2D), corresponding to a thickness equal to zero. In what follows, we work in 2-dimensional terms (direction 1 and 3 ) in order to simplify the computations (see fig. 2). The details of the computations are given in appendix B. We take $a$ to denote the elasticity tensor of the joint and $\lim a_{i j k l} / \varepsilon=\bar{a}_{i j k l}$. The limits are assumed to exist.

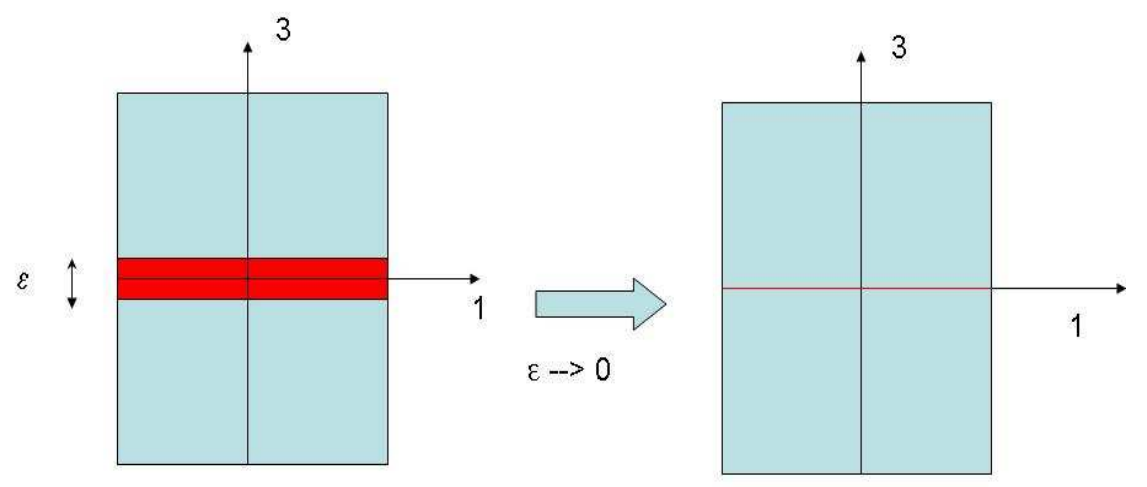

Figure 2: the principle of the asymptotic techniques in a 2-D case

We take [ ] to denote the jump along $S$. We obtain

$$
\sigma_{i 3}^{0}=\bar{a}_{i 3 i 3}\left[u_{i}^{0}\right]
$$

We find an interface law which links the stress vector to the jump in the displacement via a diagonal matrix. In our case, the terms $C_{N}$ and $C_{T}$ 
in this matrix (corresponding to the normal and tangential jumps in the displacement) are given by

$$
\left\{\begin{array}{lll}
C_{N}=\bar{C}_{3333}(\varepsilon \rightarrow 0) & \text { where } & \bar{C}_{3333}=\frac{\tilde{C}_{3333}}{\varepsilon} \\
C_{T}=\bar{C}_{1313}(\varepsilon \rightarrow 0) & \text { where } & \bar{C}_{1313}=\frac{\tilde{C}_{1313}}{\varepsilon}
\end{array}\right.
$$

Using expressions $((13),(12))$ and since the crack density scalar can be expressed in the form: $\rho=l^{2} / \varepsilon L_{0}$, where $L_{0}$ denotes the joint length, it can be established that the normal and tangential joint stiffnesses read:

$$
\left\{\begin{aligned}
C_{N} & =\frac{L_{0}}{2 C(1+D \cos 2 \phi) l^{3}} \\
C_{T} & =\frac{L_{0}}{4 C(1-D \cos 2 \phi) l^{3}}
\end{aligned}\right.
$$

The present model takes the evolution of the micro-crack into account by taking a variable crack half length $l$ depending on the load. For the following applications and for the sake of simplicity, we first assume that the half length $l$ depends only on the predominant tangential stress $\tau$ and we neglect its dependance on the normal stress. For its evolution, we assume that $l$ remains constant $\left(l=\xi_{1} L_{0}\right.$, where $\xi_{1}$ is a scalar such as $\left.0<\xi_{1}<1\right)$ until a certain value $\tau_{0}$ of the shear stress has been reached. From this value, the crack half length $l$ evolves linearly with respect to the shear stress $\tau$ up to a second value of the crack length $\xi_{2} L_{0}\left(\xi_{1}<\xi_{2}<1\right)$ reached at the maximum shear stress value $\tau_{\max }$. The following figure (Fig. 3) describes the evolution of the half crack length with respect to the shear stress $\tau$ :

The first step (where $l$ is constant) corresponds to a stable state of the interface material in which crack propagation occurs. The second step (where $l$ evolves linearly as a function of the shear stress $\tau$ ) includes the crack propagation, which leads to the failure of the interface.

The values of the shear stresses $\tau_{0}$ and $\tau_{\max }$ are fixed in advance, based on experimental 'stress-displacement' diagrams obtained on various masonries subjected to shear conditions. The values of the crack evolution law parameters $\left(\xi_{1}\right.$ and $\left.\xi_{2}\right)$ result from the minimization of the difference between the numerical and experimental 'stress-displacement' diagrams. 


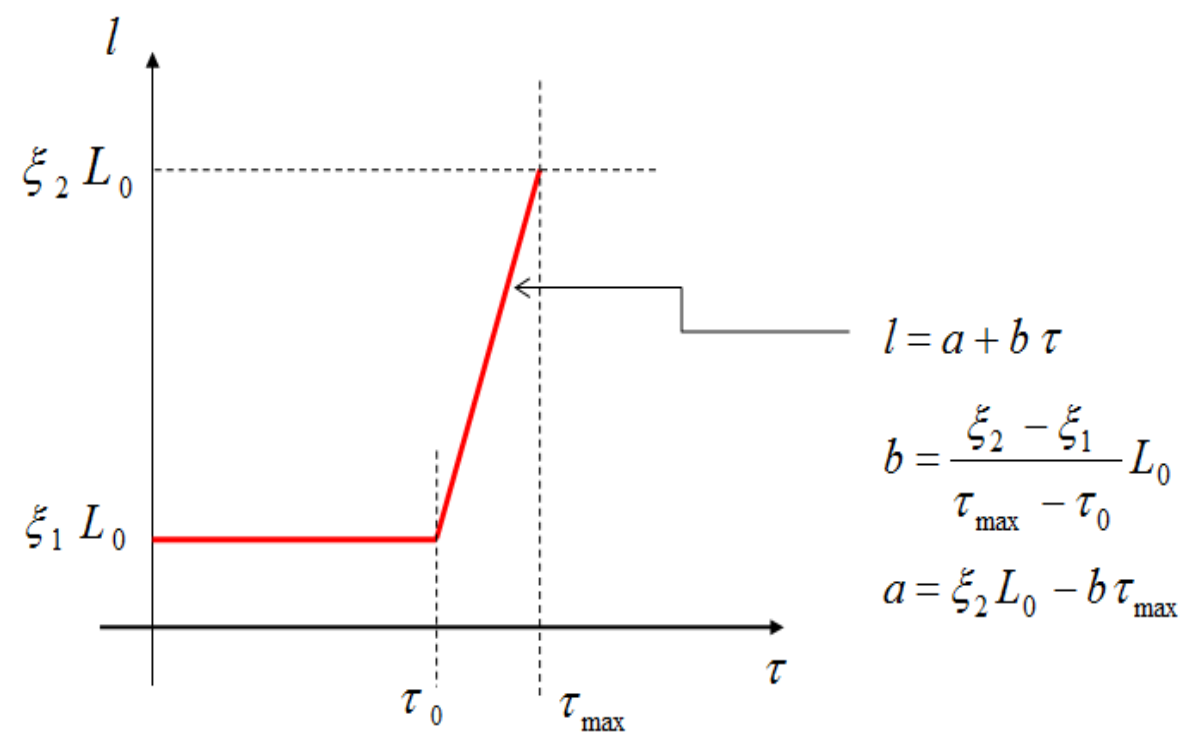

Figure 3: Function describing the evolution of the crack half length with respect to the applied shear stress

\section{Results and discussion}

The numerical evaluation of the model described above is restricted to the case of 2D plane stress in masonry structures subjected to shear loading. The obtained numerical results are then compared with experimental data available in the literature [36].

In this paper, two cases are considered: a triplet of full bricks and a triplet of hollow bricks, both subjected twice to the same nominal loading (with no lateral confinement) as shown in figure (4). Table (1) lists the material properties of the mortar and full bricks used to simulate a triplet of full bricks 


\begin{tabular}{ll}
\hline Young's moduli (MPa) of full brick & 9438 \\
Poisson ratio of full brick & 0.13 \\
Young's moduli (MPa) of hollow brick & 6059 \\
Poisson ratio of hollow brick & 0.13 \\
Young's moduli (MPa) of mortar & 4000 \\
Poisson ratio of mortar & 0.3 \\
\hline
\end{tabular}

Table 1: Mechanical properties of the three-fold masonry constituents [36]

under the shear loading conditions described (Fig. (4)). The properties of the full bricks given in this table were based on experiments by Fouchal et al [36]. Those of the hollow bricks were deduced using the self-consistent estimate $[21,22]$, based on the fact that the porosity ratio of the bricks equals $30 \%$. These homogenized properties are in good agreement with the results of experiments performed in [36].

Case of the triplet of full bricks. The properties of the undamaged material are those of the equivalent behavior of the mortar and full brick sandwich (see table 1), assuming the existence of an equal volume fraction between these two materials $\left(f_{m}=f_{b}=0.5\right)$. In the case of this sandwich configuration, the constants $\alpha^{i}(23,25), \beta^{i}(32,33), \gamma^{i}(37)$ are equal to:

$\alpha_{b}=-0.229, \alpha_{m}=0.229, \beta_{b}=-0.884, \beta_{m}=-0.115, \gamma_{b}=1.461, \gamma_{m}=0.538$

The compliance tensor $\tilde{S}^{0}$ therefore reads:

$$
10^{-4}\left(\begin{array}{rrrrrr}
1.478 & -0.271 & -0.348 & 0 & 0 & 0 \\
-0.271 & 1.478 & -0.348 & 0 & 0 & 0 \\
-0.348 & -0.348 & 1.639 & 0 & 0 & 0 \\
0 & 0 & 0 & 4.444 & 0 & 0 \\
0 & 0 & 0 & 0 & 4.444 & 0 \\
0 & 0 & 0 & 0 & 0 & 3.499
\end{array}\right)
$$

The engineering constants defining this undamaged homogeneous material (HEM-1) are therefore:

$$
\begin{gathered}
\tilde{E}_{1}^{0}=\tilde{E}_{2}^{0}=6762.57 \mathrm{MPa}, \tilde{G}_{12}^{0}=5714.57 \mathrm{MPa}, \text { and } \tilde{\nu}_{12}^{0}=0.183 \\
\tilde{E}_{3}^{0}=6099.53 \mathrm{MPa}, \tilde{G}_{13}^{0}=\tilde{G}_{23}^{0}=4497.12 \mathrm{MPa}, \text { and } \tilde{\nu}_{13}^{0}=\tilde{\nu}_{23}^{0}=0.236
\end{gathered}
$$


To evaluate the engineering constants defining the homogeneous damaged material (HEM-2), we need to determine the coefficients $C$ and $D(11)$ which are independent of the crack length parameter $2 l$ :

$$
C=4.22310^{-4} \text { and } D=0.0258
$$

Lastly, the normal and tangential stiffness of the interface are given by the following expressions, respectively:

$$
C_{N}=242365 / l^{3}\left(N / m m^{2}\right) \text { and } C_{T}=255199 / 2 l^{3}\left(N / m m^{2}\right)
$$

The experimental responses (blue dashed and pink dashed-dotted curves) are used to identify the parameters $\xi_{1}$ and $\xi_{2}$ as follows:

$$
\left\{\begin{array}{l}
\text { for the blue dashed curve: } \quad \xi_{1} L_{0}=1.47 \mu \mathrm{m}, \quad \xi_{2} L_{0}=1.484 \mu \mathrm{m} \\
\text { for the pink dashed-dotted curve: } \quad \xi_{1} L_{0}=1.397 \mu \mathrm{m}, \quad \xi_{2} L_{0}=1.47 \mu \mathrm{m}
\end{array}\right.
$$

The shear stresses values taken from the experimental 'stress-displacement' curves and serving to construct a simplified 'two-slope' crack evolution law, as depicted in Figure (3), are:

$$
\left\{\begin{array}{l}
\text { for the blue dashed curve: } \tau_{0}=0.7 \mathrm{MPa}, \tau_{\max }=0.83 \mathrm{MPa} \\
\text { for the pink dashed-dotted curve: } \tau_{0}=1.26 \mathrm{MPa}, \tau_{\max }=1.6 \mathrm{MPa}
\end{array}\right.
$$

Case of the triplet of hollow bricks. With this triplet of hollow bricks, the coefficients $\alpha^{i}, \beta^{i}$ and $\gamma^{i}, i=b, m$, are equal to:

$\alpha_{b}=-0.168, \alpha_{m}=0.168, \beta_{b}=-0.717, \beta_{m}=-0.282, \gamma_{b}=1.270, \gamma_{m}=0.729$

The compliance tensor $\tilde{S}^{0}$ of this homogeneous undamaged material HEM-1 is written as follows:

$$
10^{-4}\left(\begin{array}{rrrrrr}
1.973 & -0.396 & -0.456 & 0 & 0 & 0 \\
-0.396 & 1.973 & -0.456 & 0 & 0 & 0 \\
-0.456 & -0.456 & 1.985 & 0 & 0 & 0 \\
0 & 0 & 0 & 5.115 & 0 & 0 \\
0 & 0 & 0 & 0 & 5.115 & 0 \\
0 & 0 & 0 & 0 & 0 & 4.740
\end{array}\right)
$$


The engineering constants are therefore:

$$
\begin{gathered}
\tilde{E}_{1}^{0}=\tilde{E}_{2}^{0}=5066.57 \mathrm{MPa}, \tilde{G}_{12}^{0}=4219.43 \mathrm{MPa}, \text { and } \tilde{\nu}_{12}^{0}=0.2 \\
\tilde{E}_{3}^{0}=5037.2 \mathrm{MPa}, \tilde{G}_{13}^{0}=3910.1 \mathrm{MPa}, \text { and } \tilde{\nu}_{13}^{0}=0.23
\end{gathered}
$$

The constants $C$ and $D(11)$ used to evaluate the components of the COD tensor $B$ are:

$$
C=5.23210^{-4} \text { and } D=14.5310^{-4}
$$

The normal and tangential stiffness of the interface sandwiched between mortar and hollow brick is given by the following expressions:

$$
C_{N}=200396 / l^{3}\left(N / m^{2}\right) \text { and } C_{T}=200980 / 2 l^{3}\left(N / m^{2}\right)
$$

The blue dashed experimental curve, which shows that failure occurs at the interface and not across the thickness of the mortar or across the hollow brick, makes it possible to identify the following two parameters $\xi_{1}$ and $\xi_{2}: \xi_{1} L_{0}=1.60 \mu \mathrm{m}$ and $\xi_{2} L_{0}=1.61 \mu \mathrm{m}$, obtained for the shear stresses $\tau_{0}=0.63 M P a$ and $\tau_{\max }=0.67 M P a$, respectively. Fig.(4, (a)) shows the initial geometrical configuration of the three-fold masonry structure. The deformation and the 'stress-displacement' relation of the triplet of full bricks under shear loading conditions are shown in Fig.((4, (b)) and Fig.(5), respectively. The numerical results obtained with the present model match the experimental data (curves denoted by $1-f$ and $2-f$ ) and are in good agreement particularly with the test data obtained in 1 (curve 1-f).

Fig.(6) shows the numerical results and the experimental data obtained on a triplet of hollow bricks under shear loading conditions. In the case of the experimental data denoted by $1-\mathrm{h}, 2-\mathrm{h}$ and 3 -h, the failure occurred only across the mortar phase (figure (7), (b)) or the brick and mortar phases (figure $(7),(\mathrm{a}))$. These experimental tests $(1-\mathrm{h}, 2$-h and 3 -h) do not show the failure occurring across the brick/mortar interface and were therefore not used to obtain our numerical results. The experimental data denoted 4-h show the ability of the present model to predict the failure process across the brick/mortar interface.

The numerical results provided by the present model accurately describe the 'stress-displacement' relationship in a masonry structure where the failure occurs at the brick-mortar interface. 


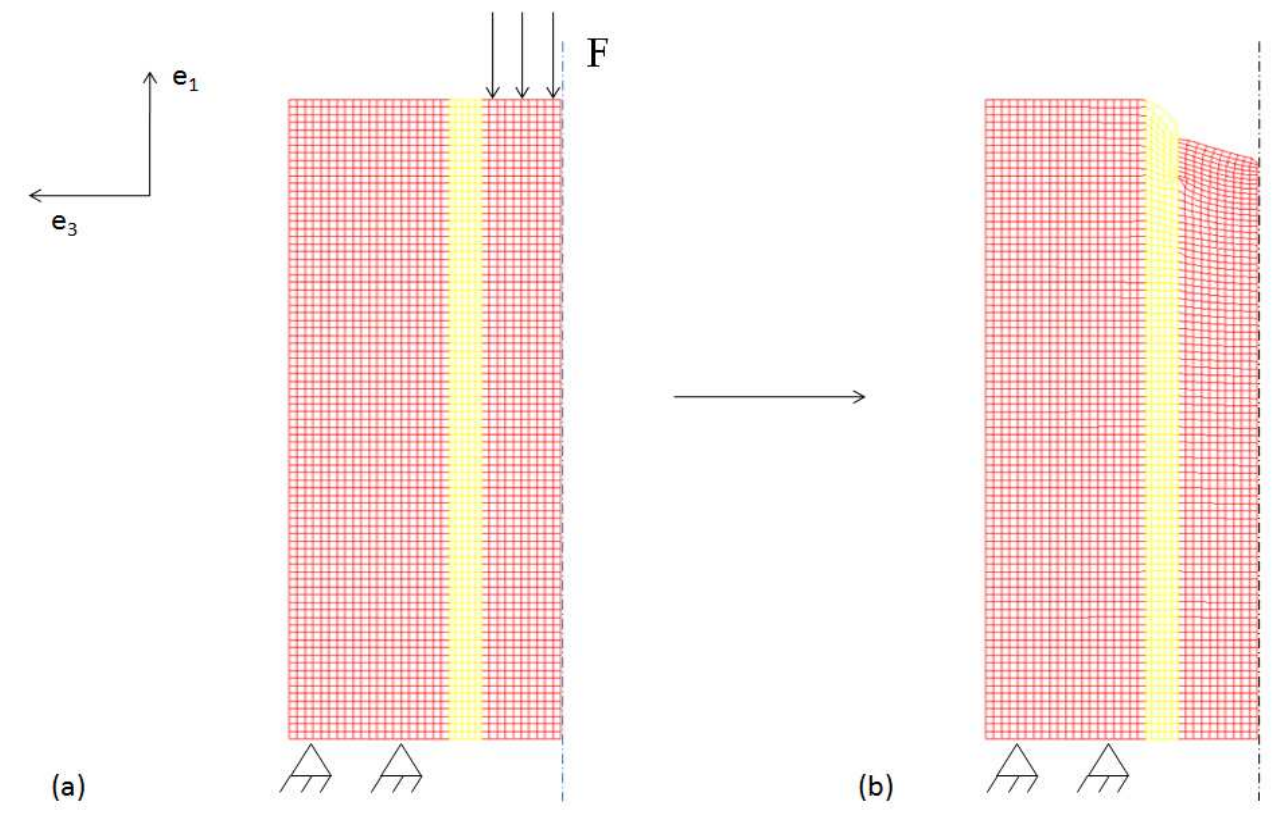

Figure 4: Initial geometrical configuration and loading conditions imposed on the triplet of full bricks (a), deformation of the triplet of full bricks in a shear test (b)

\section{Conclusion}

The multi-scale model presented here was successfully used to simulate the experimental tests in which failure occurred at the brick/mortar interface presented in [36], which provided the coefficients required to model the interface, namely the stiffness parameters and the length of the micro-cracks. The model is sensitive to these characteristics but the results obtained are in line with the experimental data.

The present method was also tested on a more complex structure presented in the literature (the case of a full wall subjected to diagonal compression loading [7]). The results obtained show that the model presented here can be used to describe the behavior of structures of this kind.

From the practical point of view, an optimization routine is needed to sys- 


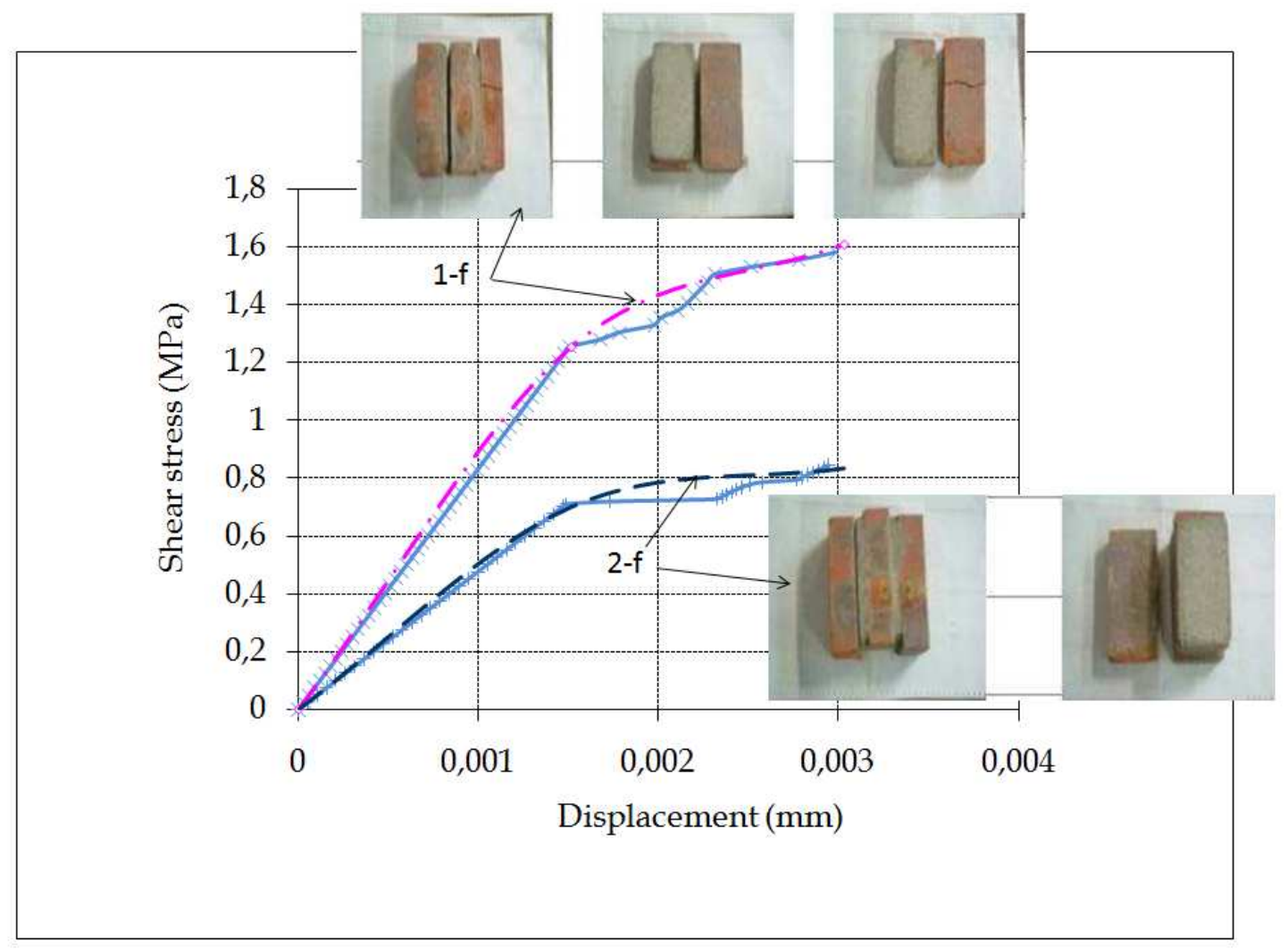

Figure 5: Stress-displacement diagrams of the triplet of full bricks under shear, numerical and experimental results.

tematically determine the values of the parameters describing the evolution of the crack length $\left(\xi_{1}\right.$ and $\left.\xi_{2}\right)$. This idea will be applied to more complex masonry structures in a future study.

It is proposed in the future to establish an optimization routine for systematically determining the values of the parameters describing the evolution of the crack length $\left(\xi_{1}\right.$ and $\left.\xi_{2}\right)$, which have depended up to now only on the shear stress. In a future study, it is plained to also examine the dependence on the normal stress.

More complex structures such as modern masonry buildings will also be 


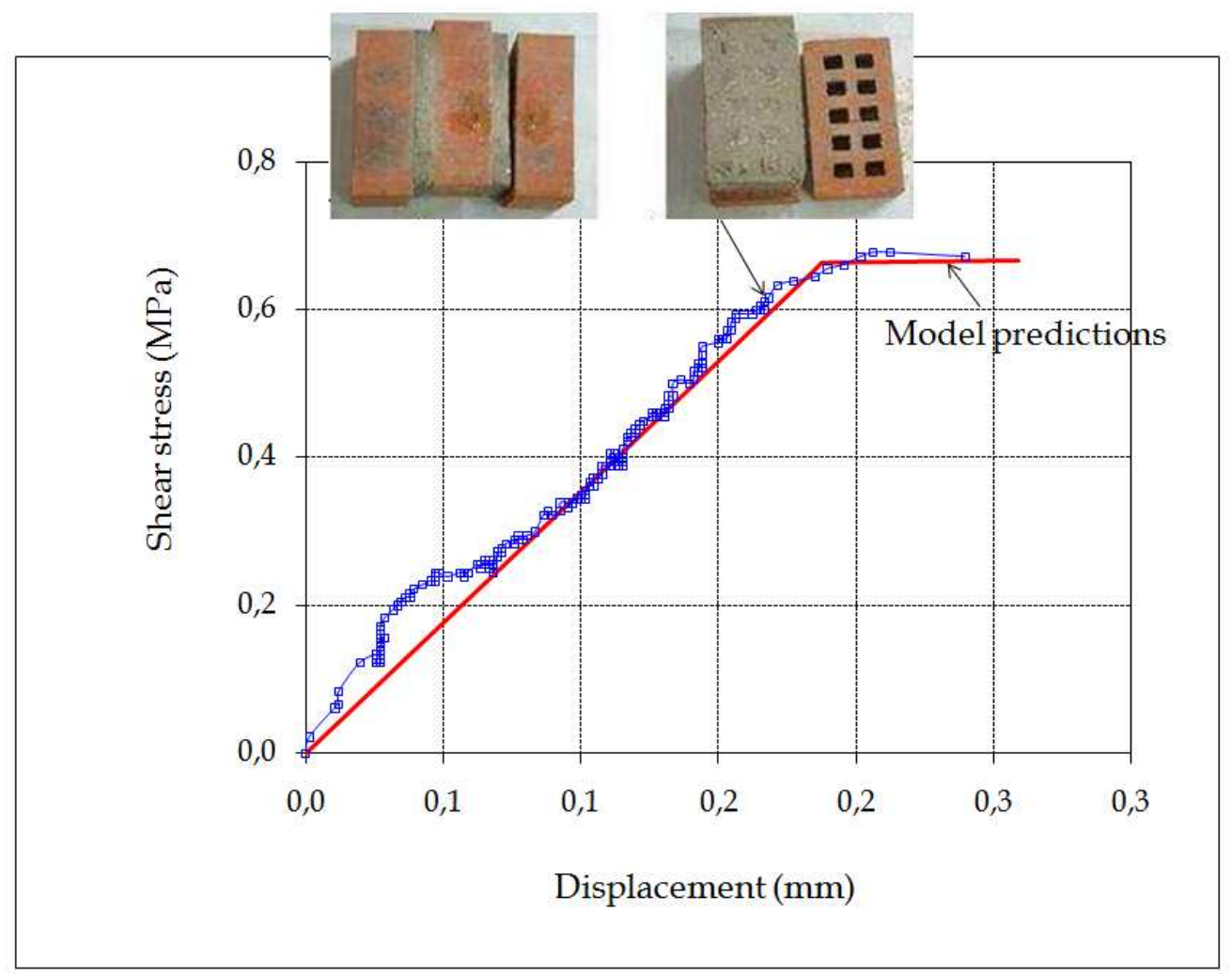

Figure 6: Stress-displacement diagrams of the triplet of hollow bricks under shear loading conditions: numerical and experimental results.

studied to improve the model presented here, by accounting more efficiently for the evolution of the micro-cracks more efficiently into account, in particular.

\section{References}

[1] Orduna A., Lourenço P.B., Three-dimensional limit analysis of rigid blocks assemblages. Part II: Load-path following solution procedure and validation, Int. J. of Sol. and Struct., 42, 5161-5180 (2005). 
(a)
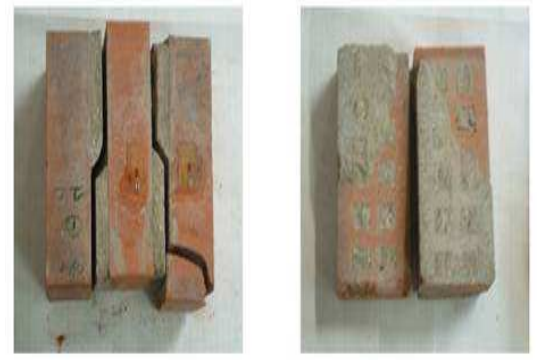

Figure 7: Experimental data on a masonry structures composed of hollow bricks under shear stress loading conditions. Failure across the mortar and brick phases (a), across the mortar phase alone (b)

[2] Lourenço P.B., Barros J.O., Olveira J.T., Shear testing of stack bonded masonry, Construction and Building Materials., 18, 125-132 (2004).

[3] Anthoine A., Magonette G. and Magenes G., Shear-compression testing and analysis of brick masonry walls, in G. Duma (ed), Proc. 10th European conf. Earthquake eng., Vol. 3, Balkema, Rotterdam, pp. 1657-1662 (1995).

[4] Benedetti D., Benzoni G.M., A numerical model for seismic analysis of masonry building: Experimental Correlations, Earthquake Eng. Struct. dyn. 12, 817-831 (1984).

[5] Lotfi H. R., Shing P. B., An appraisal of smeared crack models for masonry shear wall analysis, Comput. Struct. 41, 413-425 544 91).

[6] Gabor A., Ferrier E., Jacquelin E. and Hamelin P., Analysis of the inplane shear behaviour of FRP reinforced hollow brick masonry walls, Struct. Eng. Mech., 19, 237-260, (2005).

[7] Gabor A., Bennani A., Jacquelin E. and Lebon F., Modelling approaches of the in-plane shear behaviour of unreinforced and FRP 548 engthened masonry panels, Comp. Struct., 74, 277-288, 2006.

[8] Raous M., Cangémi L. and Cocou M., A consistent model coupling adhesion, friction and unilateral contact, Comp. Methods. Appl. Mech. Engn. 177, 383-399 (1999). 
[9] Monerie Y. and Raous M., A model coupling adhesion to friction for the intercation between a crack and a fiber/matrix interface, 552 .M.M. 80, 205-209 (2000).

[10] Frémond M., Adhérence des solides, J. Mec. Theor. Appl., 6, 383-407 (1987).

[11] Lebon F., Ronel-Idrissi S., Asymptotic studies of Mohr-Coulomb and Drucker-Prager soft thin layers, International Journal of 556 el and Composite Structures, Vol. 4, pp. 133-148, 2004

[12] Lebon F., Rizzoni, R., Ronel-Idrissi, S. Analysis of non-linear soft thin interfaces, Computers and Structures, Vol 82, pp 558 9-1938, 2004

[13] Lebon F., Ronel-Idrissi, S. First order numerical analysis of linear thin layers, ASME Journal of Applied Mechanics, Vol. 74, pp. 824-828, 2007

[14] Lebon F., Rizzoni, R., Asymptotic study of soft thin layer: the non convex case, Mechanics of Advanced Materials and Structures, Vol. 15, pp. 12-20, 2008

[15] Mauge C., Kachanov M., Effective elastic properties of an anisotropic material with arbitrarily oriented interacting cracks, J. 564 h. Phys. Solids, Vol. 42, pp. 561-584, 1994

[16] Kachanov M., Effective elastic properties of cracked solids: critical review of some basic concepts, Appl. Mech. Rev. 45 (8), pp 566 -335, 1992

[17] Kachanov M., Elastic solids with many cracks and related problems, Advances in Applied Mechanics (ed. J. Hutchinson and T. Wu), 568 30, pp 259-445, Academic Press, New-York, 1993

[18] Chaboche J-L, Suquet P., Besson J., Endommagement et changement d'échelle, in: Bornert M., Bretheau T., Gilormini P. (Eds), 570 ogénéisation en mécanique des matériaux, tome2, Hermes Science, Chapter 3, pp. 91-146, 2001

[19] Lekhnitsky S. G., Theory of elasticity of an anisotropic elastic body, OGIZ (in Russian) English Transl.: Mir Publishers, 1961 
[20] Savin G. N., Stress concentration around holes, Gostekhteoretzdat (in Russian). English transl.: Pergamon Press, 1960

[21] Hutchinson J., Bounds and self-consistent estimates for creep of polycrystalline materials, Proc. Roy. Soc. London A348, pp. 574 -127, 1976

[22] Berveiller M., Zaoui A., An extension of the self-consistent scheme to plastically-flowing polycrystals, J. Mech. Phys. Solids 576 pp. 325-344, 1979

[23] Suquet P., Bornert M., Calcul tensoriel et élasticité, in: Bornert M., Bretheau T., Gilormini P. (Eds), Homogénéisation 578 mécanique des matériaux, tome 2, Hermes Science Publications, chapter 5, pp. 171-202, 2001

[24] Milani E., Milani G., Tralli A., Limit analysis of masonry vaults by means of curved shell finite elements and homogenization, 582 ernational Journal of Solids and Structures, Vol. 45, Issue 20, pp. 5258-5288, 2008

[25] Dallot J., Sab K., Godet O., Experimental validation of a homogenized plate model for the yield design of masonry walls, Comptes 584 dus Mécanique, Vol. 336, Issue 6, pp. 487-492, 2008

[26] Wei X., Hao H., Numerical derivation of homogenized dynamic masonry material properties with strain rate effects, International 586 rnal of Impact Engineering, in press, 2008

[27] Wu C., Hao H., Numerical derivation of averaged material properties of hollow concrete block masonry, Engineering Structures, Vol. 588 Issue 3, pp. 870-883, 2008

[28] Kawa M., Pietruszczak S., Shieh-Beygi B., Limit states for brick masonry based on homogenization approach, International Journal 590 Solids and Structures, Vol. 45, Issues 3-4, pp. 998-1016, 2008

[29] Dallot J., Sab K., Limit analysis of multi-layered plates. Part I: The homogenized Love-Kirchhoff model, Journal of the Mechanics 592 Physics of Solids, Vol. 56, Issue 2, pp. 561-580, 2008

[30] Falsone G., Lombardo M., Stochastic representation of the mechanical properties of irregular masonry structures, International $594 \mathrm{rnal}$ of Solids and Structures, Vol. 44, Issues 25-26, pp. 8600-8612, 2007 
[31] Cecchi A., Milani G., Tralli A., Out-of-plane loaded CFRP reinforced masonry walls: Mechanical characteristics by homogenization 596 cedures, Composites Science and Technology, Vol. 65, Issue 10, pp. 14801500,2005

[32] Carbone V.I., Codegone M., Homogenization process of stratified masonry, Mathematical and Computer Modelling, Vol. 42, Issues 3-4, 598 375-380, 2005

[33] Cluni F., Gusella V., Homogenization of non-periodic masonry structures, International Journal of Solids and Structures, Vol. 41, 600 ue 7, pp. 1911-1923, 2004

[34] Sab K., Yield design of thin periodic plates by a homogenization technique and an application to masonry walls, Comptes Rendus 602 anique, Vol. 331, Issue 9, pp. 641-646, 2003

[35] Marfia S., Sacco E., Modeling of reinforced masonry elements, International Journal of Solids and Structures, Vol. 38, Issues 604 25, pp. 4177-4198, 2001

[36] Fouchal F., Lebon F., Titeux I., Contribution to the modelling of interfaces in masonry construction, Construction and Building 607 erials, Vol. 23 (6), pp. 2428-2441 (2009).

[37] Sacco E., A nonlinear homogenization procedure for periodic masonry, European Journal of Mechanics A/Solids, Vol. 28 (2), pp. $609-222$ (2009)

[38] Sejnoha J., Sejnoha M., Zeman J., Sykora J., Vorel J., Mesoscopic study on historic masonry, Structural Engineering and Mechanics, 612 . 30, Issue 1, pp. 99-117, 2008

[39] Boutin C., Microstructural effects in elastic composites, International Journal of Solids and Structures, Vol. 33, pp. 1023-1051, 6146

[40] Sanchez P., In: Homogenization Techniques for composites media, Lecture Note in Physics, 272, pp. 122-189, Springer, Berlin, 6165 


\section{Appendix A: Effective properties of the undamaged stratified brick/mortar: HEM-1}

Axial tension. The brick/mortar laminate is subjected to an axial stress:

$$
\bar{\sigma}=\bar{\sigma}_{33} e_{3} \otimes e_{3}
$$

In this case, relation (3) involves the equalities:

$$
\begin{aligned}
& \bar{\varepsilon}_{11}=\bar{\varepsilon}_{22}=\tilde{S}_{1133}^{0} \bar{\sigma}_{33} \\
& \bar{\varepsilon}_{33}=\tilde{S}_{3333}^{0} \bar{\sigma}_{33} \\
& \bar{\varepsilon}_{23}=\bar{\varepsilon}_{13}=\bar{\varepsilon}_{12}=0
\end{aligned}
$$

In each phase, we assume the local stress induced by the macroscopic loading to be transversely isotropic with the form:

$$
\sigma^{i}=\bar{\sigma}_{33}\left(e_{3} \otimes e_{3}+\alpha^{i}\left(e_{1} \otimes e_{1}+e_{2} \otimes e_{2}\right)\right),
$$

where $\alpha^{i}(i=b, m)$ are real constants determined so that (4) and (5) are fulfilled. The average stress $\bar{\sigma}=\sum_{i=b, m} f^{i} \sigma^{i}$ means that $\sum_{i=b, m} f^{i} \alpha^{i}=0$. The real constant $\alpha^{m}$ can then be expressed as a function of the primary constant $\alpha^{b}$. It reads:

$$
\alpha^{m}=-\frac{f^{b}}{f^{m}} \alpha^{b}
$$

Under the present loading conditions (20), the local deformation reads

$$
\varepsilon_{k j}^{i}=S_{k j l n}^{i} \sigma_{l n}^{i}=\left(S_{k j 33}^{i}+\alpha^{i}\left(S_{k j 11}^{i}+S_{k j 22}^{i}\right)\right) \bar{\sigma}_{33}, \quad i=b, m
$$

Applying relations (5) and (24) to the couplet $(1,1)$ gives the constant $\alpha^{b}$, which reads:

$$
\alpha^{b}=\frac{f_{m}\left(S_{1133}^{m}-S_{1133}^{b}\right)}{f_{b}\left(S_{1111}^{m}+S_{1122}^{m}\right)+f_{m}\left(S_{1111}^{b}+S_{1122}^{b}\right)}
$$

To identify the effective compliance $\tilde{S}_{1133}^{0}$ of the material HEM-1, we then use the relations:

$$
\begin{cases}\bar{\varepsilon}_{11}=\tilde{S}_{1133}^{0} \bar{\sigma}_{33} & \text { (a) } \\ \bar{\varepsilon}_{11}=\sum_{i=b, m} f^{i} \varepsilon_{11}^{i}=\sum_{i=b, m} f^{i}\left(S_{1133}^{i}+\alpha^{i}\left(S_{1111}^{i}+S_{1122}^{i}\right)\right) \bar{\sigma}_{33} & 6680 x(b)\end{cases}
$$

and $\tilde{S}_{1133}^{0}$ therefore reads:

$$
\tilde{S}_{1133}^{0}=\sum_{i=b, m} f^{i}\left[S_{1133}^{i}+\alpha^{i}\left(S_{1111}+S_{1122}^{i}\right)\right]
$$


We proceed in a similar way with the macroscopic strain component $\bar{\varepsilon}_{33}$ in order to determine the compliance component $\tilde{S}_{3333}^{0}$ as follows:

$$
\tilde{S}_{3333}^{0}=\sum_{i} f^{i}\left[S_{3333}^{i}+\alpha^{i}\left(S_{3311}^{i}+S_{3322}^{i}\right)\right]
$$

Axisymmetric loading. It is now proposed to subject the laminate to the following axisymmetric loading

$$
\bar{\sigma}=\bar{\sigma}_{33}\left(e_{3} \otimes e_{3}-\frac{1}{2}\left(e_{1} \otimes e_{1}+e_{2} \otimes e_{2}\right)\right)
$$

Based on the overall constitutive relation (3), the macroscopic strain response components read:

$$
\left\{\begin{array}{l}
\bar{\varepsilon}_{11}=\bar{\varepsilon}_{22}=\left(-\frac{1}{2}\left(\tilde{S}_{1111}^{0}+\tilde{S}_{1122}^{0}\right)+\tilde{S}_{1133}^{0}\right) \bar{\sigma}_{33} \\
\bar{\varepsilon}_{33}=\left(-\tilde{S}_{1133}^{0}+\tilde{S}_{3333}^{0}\right) \bar{\sigma}_{33} \\
\bar{\varepsilon}_{23}=\bar{\varepsilon}_{13}=\bar{\varepsilon}_{12}=0
\end{array}\right.
$$

The local stress induced by the macroscopic stress (29) is assumed to take the form:

$$
\left.\sigma^{i}=\bar{\sigma}_{33}\left(e_{3} \otimes e_{3}+\beta^{i}\left(e_{1} \otimes e_{1}+e_{2} \otimes e_{2}\right)\right)\right)
$$

where $\beta^{i}(i=b, m)$ are real constants determined so that the conditions specified above in (4) and (5) are met.

The average stress over the laminate gives the expression: $\sum_{i=b, m} f^{i} \beta^{i}=-\frac{1}{2}$. The constant $\beta^{m}$ can then be expressed as a function of $\beta^{b}$ as follows:

$$
\beta^{m}=\frac{-1 / 2-f^{b} \beta^{b}}{f^{m}}
$$

It is now only necessary to express the constant $\beta^{b}$.

The first continuity condition (4) is ensured by the assumed form of the local stress (31), whatever are the values of the constants $\beta^{i}$. The continuity condition (5) is used to express $\beta^{b}$ :

$$
\beta^{b}=\frac{f_{m}\left(S_{1133}^{m}-S_{1133}^{b}\right)-\frac{1}{2}\left(S_{1111}^{m}+S_{1122}^{m}\right)}{f_{1}\left(S_{1111}^{m}+S_{1122}^{m}\right)+f_{2}\left(S_{1111}^{b}+S_{1122}^{b}\right)}
$$

The average strain component $\bar{\varepsilon}_{11}$ given by relations $(30,(\mathrm{a}))$ and $(26,(\mathrm{~b}))$ gives a first algebraic equation for the compliance components $\tilde{S}_{1111}^{0}$ and $\tilde{S}_{1122}^{0}$ :

$$
\tilde{S}_{1111}^{0}+\tilde{S}_{1122}^{0}=2 \tilde{S}_{1133}^{0}-2 \sum_{i=b, m} f_{i}\left[S_{1133}^{i}+\beta^{i}\left(S_{1111}^{i}+S_{1122}^{i}\right)\right]
$$


Mixed load: plane traction and plane shear. In this paragraph, the macroscopic stress applied to the stratified crack-free composite reads:

$$
\bar{\sigma}=\bar{\sigma}_{12}\left(e_{1} \otimes e_{2}+e_{2} \otimes e_{1}\right)+\frac{1}{2}\left(\sigma_{11}-\sigma_{22}\right)\left(e_{1} \otimes e_{1}-e_{2} \otimes e_{2}\right)
$$

The assumed local stress reads:

$$
\sigma^{i}=\gamma^{i} \bar{\sigma}, \quad i=b, m
$$

where $\gamma^{i}(i=b, m)$ has to be determined in order to meet the continuity conditions (4) and (5). The average value of the local stress solution (36) means that $\sum_{i=b, m} f^{i} \gamma^{i}=1$. The local strain continuity condition (5) can be used to determine the real primary constant $\gamma^{b}$, which reads:

$$
\gamma^{b}=\frac{S_{1212}^{m}}{f_{b} S_{1212}^{m}+f_{m} S_{1212}^{b}}=\frac{S_{1111}^{m}-S_{1122}^{m}}{f_{b}\left(S_{1111}^{m}-S_{1122}^{m}\right)+f_{m}\left(S_{1111}^{b}-S_{1122}^{b}\right)}
$$

Based on Hooke's law, the local strain reads: $\varepsilon^{i}=S^{i} \sigma^{i}=\gamma^{i} S^{i} \bar{\sigma}$. With this kind of macroscopic load $(35)$, the couplet $(1,2)$ in relation $(3)$ can be used to identify the effective compliance component $\tilde{S}_{1212}^{0}$ as follows:

$$
\tilde{S}_{1212}^{0}=\sum_{i=b, m} f^{i} \gamma^{i} S_{1212}^{i}
$$

The average local strain components $\varepsilon_{11}$ and $\varepsilon_{22}$ in the stratified composite give the following algebraic relations:

$$
\begin{aligned}
& \tilde{S}_{1111}^{0}-\tilde{S}_{1122}^{0}=\sum_{i=b, m} f_{i} \gamma^{i}\left(S_{1111}^{i}-S_{1122}^{i}\right) \\
& \tilde{S}_{1122}^{0}-\tilde{S}_{2222}^{0}=\sum_{i=b, m} f_{i} \gamma^{i}\left(S_{2211}^{i}-S_{2222}^{i}\right)
\end{aligned}
$$

Expressions (34) and (38, (a)) are used to determine the HEM-1 compliance components $\tilde{S}_{1111}^{0}$ and $\tilde{S}_{1122}^{0}$. Relation $(38,(\mathrm{~b}))$ is then used to obtain the compliance component $\tilde{S}_{2222}^{0}$.

Longitudinal shear loading. In this case, the macroscopic stress applied is:

$$
\bar{\sigma}=\bar{\sigma}_{13}\left(e_{1} \otimes e_{3}+e_{3} \otimes e_{1}\right)+\bar{\sigma}_{23}\left(e_{2} \otimes e_{3}+e_{3} \otimes e_{2}\right)
$$

The local stress solution reads $\sigma^{i}=\delta^{i} \bar{\sigma}$, where $\delta^{i}(i=b, m)$ are such that the local stress meets the continuity conditions $((4),(5))$. The average stress 
gives the following relation between the constants $\delta^{i}: \sum_{i} f^{i} \delta^{i}=1$. The continuity of the force vector at the brick/mortar interface (condition (4)) means that: $\delta^{b}=\delta^{m}=1$. With the couplet $(2,3)$, relations (3) and (1) are used to obtain the effective compliance component $\tilde{S}_{2323}^{0}$, which reads:

$$
\tilde{S}_{2323}^{0}=\sum_{i=b, m} f^{i} S_{2323}^{i}
$$

\section{Appendix B: asymptotic analysis, elements of proof}

The idea underlying matched asymptotic expansions is to find two expansions of the displacement $u^{\varepsilon}$ and the stress $\sigma^{\varepsilon}$ in powers of $\varepsilon$, i.e., an external expansion in the case of the bodies and an internal one in that of the joint, and to combine these two expansions in order to obtain the same limit. In what follows, we work in 2-dimensional terms in order to simplify the computations. The relations obtained in the internal expansions will be expressed using values occurring in the external expansions.

External asymptotic expansions. The external expansion is a classical expansion in powers of $\varepsilon$

$$
\begin{aligned}
u^{\varepsilon}\left(x_{1}, x_{3}\right) & =u^{0}\left(x_{1}, x_{3}\right)+\varepsilon u^{1}\left(x_{1}, x_{3}\right)+\ldots, \\
e_{i j}\left(u^{\varepsilon}\right)\left(x_{1}, x_{3}\right) & =e_{i j}^{0}+\varepsilon e_{i j}^{1}+\ldots, \\
e_{i j}^{l} & =\frac{1}{2}\left(\frac{\partial u_{i}^{l}}{\partial x_{j}}+\frac{\partial u_{j}^{l}}{\partial x_{i}}\right), \\
\sigma_{i j}^{\varepsilon}\left(x_{1}, x_{2}\right) & =\sigma_{i j}^{0}\left(x_{1}, x_{3}\right)+\varepsilon \sigma_{i j}^{1}\left(x_{1}, x_{3}\right)+\ldots
\end{aligned}
$$

Internal asymptotic expansions. In the internal expansions, we perform a rescaling of the second variable. Let $y_{3}=\frac{x_{3}}{\varepsilon}$. The internal expansion gives

$$
\begin{aligned}
u^{\varepsilon}\left(x_{1}, x_{3}\right) & =v^{0}\left(x_{1}, y_{3}\right)+\varepsilon v^{1}\left(x_{1}, y_{3}\right)+\ldots, \\
e_{i j}\left(u^{\varepsilon}\right)\left(x_{1}, y_{3}\right) & =\varepsilon^{-1} e_{i j}^{-1}+e_{i j}^{0}+\varepsilon e_{i j}^{1}+\ldots, \\
e_{11}^{l} & =\frac{\partial v_{1}^{l}}{\partial x_{1}}, \\
e_{33}^{l} & =\frac{\partial v_{2}^{l+1}}{\partial y_{3}}, \\
e_{13}^{l} & =\frac{1}{2}\left(\frac{\partial v_{2}^{l}}{\partial x_{1}}+\frac{\partial v_{1}^{l+1}}{\partial y_{3}}\right), \\
\sigma_{i j}^{\varepsilon}\left(x_{1}, y_{3}\right) & =\varepsilon^{-1} \tau_{i j}^{-1}\left(x_{1}, y_{3}\right)+\tau_{i j}^{0}\left(x_{1}, y_{3}\right)+\varepsilon \tau_{i j}^{1}\left(x_{1}, y_{3}\right)+\ldots, \\
\sigma_{i j, j}^{\varepsilon} & =\sum_{l=-2}^{\infty} \varepsilon^{l}\left(\frac{\partial \tau_{i 1}^{l}}{\partial x_{1}}+\frac{\partial \tau_{i 3}^{l+1}}{\partial y_{3}}\right) .
\end{aligned}
$$


Conventionally

$$
v^{l}=0, l<0, \tau^{l}=0, l<-1 .
$$

Continuity conditions. The third step in this method consists in combining the two expansions. We adopted some intermediate lines defined by $x_{3}=$ $\left.\pm \theta \varepsilon^{t}, 0<t<1, \theta \in\right] 0,+\infty\left[\right.$. When $\varepsilon$ tends to zero, $x_{3}$ tends to $0^{ \pm}$and $y_{3}=x_{3} / \varepsilon$ tends to $\pm \infty$. The principle of the method consists in assuming that the two expansions both give the same asymptotic limits, that is

$$
\begin{array}{lll}
\text { (i) } & v^{0}\left(x_{1}, \pm \infty\right) & =u^{0}\left(x_{1}, 0^{ \pm}\right) \\
\text {(ii }) & \tau^{-1}\left(x_{1}, \pm \infty\right) & =0 \\
\text { (iii) } \quad \tau^{0}\left(x_{1}, \pm \infty\right) & =\sigma^{0}\left(x_{1}, 0^{ \pm}\right) .
\end{array}
$$

At order zero, these conditions give the continuity of the displacements $\left(u^{0}\right.$ and $\left.v^{0}\right)$ and stresses $\left(\sigma^{0}\right.$ and $\left.\tau^{0}\right)$ along the surface $S$.

Elasticity of the thin layer. We take $a$ to denote the elasticity tensor of the joint and $\lim a_{i j k l} / \varepsilon=\bar{a}_{i j k l}$. This limit is assumed to exist.

$$
\begin{aligned}
\tau_{i j}^{-1} & =0 \\
\tau_{i j}^{0} & =\bar{a}_{i j k l} e_{k l}^{-1}
\end{aligned}
$$

in the thin layer.

Equilibrium. The equilibrium at -2 gives

$$
\frac{\partial \tau_{i 3}^{-1}}{\partial y_{3}}=0
$$

The equilibrium at -1 gives

$$
\frac{\partial \tau_{i 2}^{0}}{\partial y_{3}}=0
$$

The stress vector does not depend on the second variable in the thin layer. We add the connectivity conditions

$$
\begin{aligned}
v_{i}^{0} & =u_{i}^{0}\left(x_{1}, 0\right) \\
\tau_{i 3}^{0} & =\sigma_{i 3}^{0}\left(x_{1}, 0\right)
\end{aligned}
$$


Using the constitutive equations, we have

$$
\tau_{i 3}^{0}=\bar{a}_{i 2 i 2} \frac{\partial v_{i}^{0}}{\partial y_{3}} \quad \text { for } i=1,3 .
$$

By integration (the stress is constant) into $[0,1]$.

$$
\tau_{i 3}^{0}=\bar{a}_{i 3 i 3}\left[v_{i}^{0}\right]
$$

Using the connection method, we obtain

$$
\sigma_{i 3}^{0}=\bar{a}_{i 3 i 3}\left[u_{i}^{0}\right]
$$

